\title{
Clinical characteristics of adolescents referred for treatment of depressive disorders
}

Article

Accepted Version

Orchard, F., Pass, L., Marshall, T. and Reynolds, S. (2017) Clinical characteristics of adolescents referred for treatment of depressive disorders. Child and Adolescent Mental Health, 22 (2). pp. 61-68. ISSN 1475-3588 doi:

https://doi.org/10.1111/camh.12178 Available at https://centaur.reading.ac.uk/66022/

It is advisable to refer to the publisher's version if you intend to cite from the work. See Guidance on citing.

To link to this article DOI: http://dx.doi.org/10.1111/camh.12178

Publisher: Wiley

All outputs in CentAUR are protected by Intellectual Property Rights law, including copyright law. Copyright and IPR is retained by the creators or other copyright holders. Terms and conditions for use of this material are defined in the End User Agreement.

www.reading.ac.uk/centaur 
Central Archive at the University of Reading

Reading's research outputs online 
Clinical characteristics of adolescents referred for treatment of depressive disorders

Faith Orchard ${ }^{1}$, Laura Pass ${ }^{1}$, Tamsin Marshall ${ }^{2}$ and Shirley Reynolds ${ }^{1}$

${ }^{1}$ School of Psychology and Clinical Language Sciences, University of Reading

${ }^{2}$ Berkshire Healthcare NHS Foundation Trust

Corresponding author:

Faith Orchard, $\mathrm{PhD}$

School of Psychology and Clinical Language Sciences,

University of Reading, Reading, RG6 6AL, UK.

Telephone +44(0)118 3788579

Email: f.orchard@ reading.ac.uk

Keywords: depression, adolescence, diagnosis, comorbidity

Short Title: Clinical characteristics of adolescent depression

Word Count: 6696

Clinical characteristics of adolescent depression

Corresponding author: Orchard 


\begin{abstract}
Background: Adolescence is a period of increased risk for the development of depression. Epidemiological and clinical studies suggest that the phenomenology of depression may differ during childhood and adolescence. However, participants in these studies may not reflect depressed young people referred to routine clinical services. The aim of this paper is to describe referrals for depression to a UK routine public healthcare service for children and adolescents with mental health difficulties.

Method: This paper describes a consecutive series of adolescents $(\mathrm{N}=100$, aged 12-17 years), referred for depression to a routine public healthcare child and adolescent mental health service, in the south of England. Young people and their caregivers completed a structured diagnostic interview and self report measures of anxiety and depression. Results: Fewer than half of young people referred for depression met diagnostic criteria for a depressive disorder. The key symptoms reported by those with depression were low mood or irritability, cognitive disturbances, sleep disturbances and negative self-perceptions. Suicidal ideation was common and was considerably higher than reported in other studies. Caregiver and young person's accounts of adolescent symptoms of depression and anxiety were uncorrelated. Caregivers also reported fewer symptoms of depression in their child than adolescents themselves.

Conclusions: These data have direct relevance to the design and delivery of public mental health services for children and adolescents. However, we do not know how representative this sample is of other clinical populations in the UK or in other countries. There is a need to collect routine data from other services to assess the needs of this group of high risk adolescents.
\end{abstract}

Clinical characteristics of adolescent depression

Corresponding author: Orchard 


\section{Key Practitioner Message}

- Existing data suggests that the characteristics and symptoms of depression in children and adolescents may differ from those reported by depressed adults.

- These data may not generalise to adolescents who are referred to and assessed by the UK National Health Service for the treatment of depression.

- Of 100 adolescents referred for depression to a routine public child and adolescent mental healthcare service in the UK, fewer than half met diagnostic criteria for a depressive disorder.

- The most common symptoms of the depressed group were low mood or irritability, suicidal ideation, cognitive disturbances, sleep disturbances and negative self-perceptions.

- Suicidal ideation was much higher in all groups than reported in clinical trials.

- There are currently no guidelines in place regarding 1) how to treat adolescents who do not meet diagnostic criteria for any disorder, but are experiencing distress, impairment and often risk, and 2) how to support and manage clinical staff who are dealing with such high levels of risk.

Clinical characteristics of adolescent depression 
Low mood and depression often emerge during adolescence (Green, McGinnity, Meltzer, Ford, \& Goodman, 2005) and are highly prevalent (Costello, Erkanli, \& Angold, 2006). Nearly $6 \%$ of adolescents meet criteria for a depressive disorder at any given time (Costello et al., 2006), with the cumulative frequency of depression rising to as high as $20 \%$ by the end of adolescence (Thapar, Collishaw, Pine, \& Thapar, 2012). Experiencing depression in youth can have long-term negative impacts, and increases risk of further episodes of depression as an adult (Dunn \& Goodyer, 2006; Harrington \& Dubicka, 2001), and of developing other mental health disorders (Fergusson \& Woodward, 2002; Halperin, Rucklidge, Powers, Miller, \& Newcorn, 2010; Harrington, Fudge, Rutter, Pickles, \& Hill, 1991; Lewinsohn, Rohde, Klein, \& Seeley, 1999; Rao et al., 1995; Rudolph \& Klein, 2009). Adolescent onset depression has also been associated with educational underachievement, low income levels and unemployment (Fergusson, Boden, \& Horwood, 2007; Franko, Striegel-Moore, Thompson, Schreiber, \& Daniels, 2005; Gotlib, Lewinsohn, \& Seeley, 1998), and is a risk factor for suicidal behavior (Bridge, Goldstein, \& Brent, 2006).

Major Depressive Disorder (MDD) is defined as a cluster of specific symptoms that are associated with impairment. The Diagnostic and Statistical Manual of Mental Disorders 5 (DSM-5; APA, 2013) identifies possible 9 symptoms of depression - low mood (or in adolescents, irritability), anhedonia, suicidal ideation, sleep disturbances, fatigue, appetite disturbances, impaired concentration or ability to make decisions, psychomotor changes and negative self-perceptions. Five symptoms must be present and must include either low mood (or irritability in young people), or anhedonia. These symptoms must have been present for at least two weeks and must cause clinically significant distress or impairment in occupational, social or other important domains of life. The gold standard diagnostic interview for adolescent depression, the Kiddie-Schedule for Affective Disorders and Schizophrenia

Clinical characteristics of adolescent depression

Corresponding author: Orchard 
(KSADS; Kaufman et al., 1997), is currently still based on the DSM-IV (APA, 1994). However, changes for the diagnosis of depressive disorders in DSM-5 were minimal. The only change was that a diagnosis of major depression following grief or bereavement is no longer excluded. Two additional disorders were included, 'Disruptive Mood Dysregulation' and 'Premenstrual Dysphoric Disorder', and 'Dysthymia' was changed to 'Persistent Depressive Disorder'.

Depression is often not identified in adolescents (Leaf et al., 1996). With the exception of irritability, the features of depression are supposedly similar in adolescents and adults (Lewinsohn, Rohde, Seeley, Klein, \& Gotlib, 2003). A recent qualitative study by Midgley et al. (2015) focused on young people's experience of depression which highlighted some key features that are not part of diagnostic criteria e.g. feeling isolated. Weiss and Garber (2003) conducted a meta-analysis of research on the developmental stage of the child or adolescent and the associated phenomenology of depression and found that anhedonia, hopelessness, hypersomnia, weight gain and social withdrawal are more common in older adolescents compared with younger children.

Given the frequency and associated risks of adolescent depression, along with the wide variety of possible symptoms, it is important to understand the clinical presentation of depressed young people in order to inform assessment and treatment. Data about the characteristics of depression in young people come from epidemiological studies or from clinical trials of medication and psychotherapies. Epidemiological studies indicate that female adolescents are twice as likely as male adolescents to experience depression (Hyde, Mezulis, \& Abramson, 2008; Thapar et al., 2012). Other risk factors include having a parent that has experienced depression (Rice, Harold, \& Thapar, 2002; Tully, Iacono, \& McGue, 2008), exposure to stressful live events (Goodyer, Wright, \& Altham, 1990; Pine, Cohen,

Clinical characteristics of adolescent depression

Corresponding author: Orchard 
Johnson, \& Brook, 2002) and experiencing bullying (Hawker \& Boulton, 2000). Two thirds of depressed adolescents have at least one comorbid disorder (Ford, Goodman, \& Meltzer, 2003). Anxiety is the most common co-morbid mental health problem in young people with depression (Angold, Costello, \& Erkanli, 1999); around 75\% of young people with depression experience symptoms of anxiety (Essau \& Chang, 2009) and, almost 20\% of depressed adolescents also meet diagnostic criteria for generalized anxiety disorder (Angold \& Costello, 1993).

Treatment trials for adolescent depression also provide information about the clinical characteristics of their participants. A recent multi-site RCT for adolescent depression recruited 465 young people with depression from routine public mental health services for children and adolescents in England (Goodyer et al., 2015). On the basis of K-SADS diagnostic interviews carried out before treatment, the most common symptoms reported were sleep disturbance, low mood and cognitive disturbances. Recruitment to the Treatment of Adolescent Depression Study (TADS) trial in the U.S. was selective and over representative of females; $54.4 \%$ of participants were female (TADS, 2005). Of the adolescents with a primary diagnosis of depression, $27.4 \%$ had comorbid anxiety disorders, and $23.5 \%$ had comorbid behavior disorders. Symptoms elicited by the K-SADS diagnostic interview indicated that $29.2 \%$ of adolescents had thoughts of death or suicidal ideation in the two week period before assessment. In the Treatment of Resistant Depression in Adolescents trial (TORDIA; Brent et al., 2008) participants were adolescents who had not responded to at least 2 trials of SSRI or at least 7 sessions of CBT. This sample had a mean age of 16 years, $70 \%$ were females and $82 \%$ were "white". On the basis of the Suicidal Ideation Questionnaire (Reynolds \& Mazza, 1999), 58.5\% of the sample reported clinically significant 
suicidal ideation in the past month, and $51.7 \%$ of participants had at least one comorbid disorder.

The extent to which these data generalise to routine UK mental health services is not known. There is relatively little data currently reported about the characteristics of depressed adolescents who present for assessment and treatment. This paper presents data that was routinely collected in a public mental health service for children and adolescents in the south of England. It describes the clinical characteristics of 100 consecutive assessments following a referral for depression with the aim of helping inform service delivery and design.

\section{Methods}

\section{Participants}

Participants were a subset of young people and primary caregivers referred to a community public mental health service, which is free at the point of entry and assesses and treats children and adolescents with anxiety and depression. The service is based at the University of Reading as collaboration between the local healthcare service and the University (see Procedure for details of referral process). Of 103 patients who were invited to take part in the study, 3 declined participation in the research assessment so their data is not reported in this paper. Therefore 100 young people aged 12 to 17 years, and their primary caregiver, were recruited between November 2013 and August 2015. Caregivers who attended were usually their biological mother (79\%); there were also fathers (15\%) and other family members, e.g. step-parent, aunt, adopted mother (6\%).

\section{Procedure}

Children and adolescents were referred by primary and secondary care services. Referrals were triaged and those presenting with primarily moderate to severely impairing anxiety, depressive or obsessive-compulsive symptoms are transferred to the anxiety and

Clinical characteristics of adolescent depression

Corresponding author: Orchard 
depression service based at the University of Reading. Young people with mildly impairing symptoms of anxiety or depression are usually diverted to other services. Furthermore, young people who were felt to be in a high risk category due to suicide risk, safeguarding issues or psychosis were seen for urgent assessments and were not invited to take part in this research $(n=43)$. Young people with very significant psychosocial complexity, requiring significant multiagency liaison and multi-model treatment packages were also excluded as they were directed to a different child and adolescent mental health team designed to meet their particular needs.

Between November 2013 and August 2015, 103 young people were eligible for the standard depression assessment, and were invited to take part in this study. The study was approved by the Berkshire Local Research Ethics Committee and the University of Reading Research Ethics Committee. Adolescents aged 16-18 years provided informed consent for themselves, while adolescents aged 12-15 years required consent from their caregiver as well as providing assent themselves. Caregivers also provided written consent for themselves taking part.

Assessments were conducted on one day. As part of a generic clinical assessment the young person and their primary caregiver were interviewed separately. This included information on family structure, developmental history, education, and physical health. Risk assessments were carried out and a care plan was completed with the young person and primary caregiver. This was followed by a diagnostic assessment separately with the young person and primary caregiver, and the completion of standardized symptom questionnaires.

\section{Measures}

Demographics. As part of the routine clinical assessment, demographic information was collected including age, gender, ethnicity and socioeconomic status.

Clinical characteristics of adolescent depression

Corresponding author: Orchard 
Diagnoses. Adolescents referred to the clinic were assigned diagnoses on the basis of the Kiddie Schedule of Affective Disorders and Schizophrenia (K-SADS), a structured diagnostic interview for DSM-IV affective disorders and schizophrenia, with well-established psychometric properties (Kaufman et al., 1997). As is conventional, the interview was conducted with adolescents and caregivers separately, and diagnoses were based on the information obtained from both interviews. Assessors (psychology graduates) were trained on the standard administration and scoring of the K-SADS through verbal instruction, listening to assessment audio-recordings and participating in diagnostic consensus discussions. Competence was evaluated with reference to the assessors' ratings of a standard assessment recording. Once trained, all diagnoses were double-rated by both the assessor and a clinical psychologist. Inter-rater reliability for K-SADS diagnoses overall was $\mathrm{k}=.97$ and reliability for depression diagnosis specifically was $\mathrm{K}=1.00$.

Symptom Measures. The Mood and Feelings Questionnaire (MFQ) is a 33 item selfreport scale of depressive symptoms for adolescents which has good psychometric properties (Burleson Daviss et al., 2006). This study included adolescent (MFQ-C) and parent (MFQ-P) versions. Each symptom is rated on a 3 point scale from 0 (not true) to 2 (true). Internal consistency for the MFQ was very high (MFQ-C $\alpha=.92$; MFQ-P $\alpha=.97$ )

The Revised Child Anxiety and Depression Scale (RCADS) is a 37 item self-report scale used to assess anxiety and depression symptoms (Chorpita, Yim, Moffitt, Umemoto, \& Francis, 2000). Parent (RCADS-P) and adolescent (RCADS-C) reports of symptoms of adolescent anxiety and depression were included. The RCADS scoring has norms for respondent version (child or parent) and age of child. The measure has good construct validity and test-retest reliability (Chorpita, Moffitt, \& Gray, 2005). Internal consistency for the RCADS depression subscale was acceptable (RCADS-C-D $\alpha=.78$; RCADS-P-D $\alpha=$

Clinical characteristics of adolescent depression

Corresponding author: Orchard 
.87). Internal consistency for the RCADS total anxiety subscale was excellent (RCADS-C-

TOA $\alpha=.95 ;$ RCADS-P-TOA $\alpha=.97)$.

Caregivers' symptoms of depression, anxiety and stress. Caregivers' own symptoms were assessed using the short-version of the Depression Anxiety Stress Scale (DASS-21). This is a 21 item, self-report measure which has three scales relating to symptoms of depression, anxiety and stress (Lovibond \& Lovibond, 1995). Each scale consists of 7 items and items are scored using a scale of 0 (did not apply to me at all) to 3 (applied to me very much or most of the time). The DASS-21 has been used with non-clinical and clinical populations and the scales show good internal consistency and concurrent validity (Antony, Bieling, Cox, Enns, \& Swinson, 1998). Internal consistency for the DASS21 was excellent (DASS-21-D $\alpha=.99$; DASS-21-A $\alpha=.99$; DASS-21-S $\alpha=.99$ ).

\section{Results}

\section{Data Analysis}

There was no missing data on K-SADS diagnoses and symptoms, or on demographic information for the 100 participants. Some data was missing on self-report measures. Participants were excluded from the analysis of self-report measures if more than $25 \%$ of their data was missing. As a result, the following numbers were excluded from the relevant analyses: parent reported MFQ $(n=9)$, adolescent reported MFQ $(n=9)$, parent reported RCADS (depression subscale $n=8$; anxiety subscale $n=10$ ), adolescent reported RCADS ( $n$ $=8)$ and DASS-21 $(n=7)$.

When describing the data, frequencies and percentages are provided for categorical variables, and means and standard deviations (SDs) for continuous variables. Continuous data were screened in relation to the assumptions of parametric tests (Tabachnick \& Fidell, 2007). The distributions of caregivers' symptoms of depression and anxiety were positively skewed.

Clinical characteristics of adolescent depression

Corresponding author: Orchard 
As these variables could not be successfully transformed, confirmatory analyses were conducted by running analyses with 1000 bootstrap samples. Results were consistent, suggesting that the original analyses were robust to the violations of assumptions, so results based on the original (non-bootstrapped) analyses are presented for simplicity.

\section{Demographic Characteristics}

The mean age of the adolescents was 15.78 years $(\mathrm{SD}=1.19$; range $=12-17)$, with the majority (60\%) aged 15 or 16 years. Most (85\%) were female and white British (89\%) Other ethnicities included white and black Caribbean, Asian, Indian and Pakistani. Most families were classed as 'professional' $(78 \%)$. The mean age of males and females did not differ significantly (mean $(\mathrm{SD})$ : males $=15.97(.92)$, females $=15.72(1.24), t(95)=-.74, p=.46)$.

\section{Primary Disorder}

Forty-two of the 100 young people met criteria for a depressive disorder as the primary diagnosis on the K-SADS. Thirty seven met criteria for major depressive disorder, 3 met criteria for major depressive disorder with psychosis, and 2 participants met criteria for schizoaffective depressive disorder.

Of the remaining fifty-nine participants, 23 met criteria for an anxiety disorder as their primary diagnosis (social phobia $n=9$, generalized anxiety disorder $n=9$, anxiety disorder not otherwise specified $n=2$, separation anxiety $n=1$, obsessive compulsive disorder $n=1$, agoraphobia without panic disorder $n=1$ ). One participant met criteria for oppositional defiant disorder as their primary diagnosis. Thirty-four young people did not meet criteria for any mental health disorder.

\section{Depressive Symptoms}

The majority of adolescents that were diagnosed with a depressive disorder met criteria for 5 or 6 symptoms (respectively; 44\%, 34\%). All participants met the core criterion

Clinical characteristics of adolescent depression

Corresponding author: Orchard 
of 'low mood/irritability'. Over half of the adolescents with depression also met the additional core criterion of anhedonia. Other very common symptoms were negative self-perceptions, suicidal ideation and sleep disturbance. The frequencies of each of the depressive symptoms reported by adolescents with depression, another mental health disorder and no mental health diagnosis are presented in Table 1.

[Insert Table 1]

\section{Comorbidity}

Twenty-five percent of young people from the total sample met criteria for one or more comorbid disorder. One participant met criteria for 5 disorders, one participant met criteria for 4 disorders, one participant met criteria for 3 disorders, and 22 participants met criteria for 2 disorders. Comorbid diagnoses included social anxiety disorder $(n=15)$, generalized anxiety disorder $(n=10)$, panic disorder with agoraphobia $(n=2)$, panic disorder ( $n=1)$, specific phobia $(n=1)$, anxiety disorder not otherwise specified $(n=1)$, major depressive disorder ( $n=1$, in the presence of a primary anxiety disorder). Of the adolescents that met criteria for a primary diagnosis of depression, $54 \%$ had comorbid disorders. These included social anxiety disorder (32\%), generalized anxiety disorder (14\%), panic disorder (3\%), specific phobia (3\%) and anxiety disorder not otherwise specified (3\%).

\section{Disorders and Age/Gender}

There was no difference in age between those that did and did not meet criteria for any disorder, $t(95)=-1.95, p=.06$, and no association between presence of any diagnosis and gender, $\chi^{2}(1)=.28, p=.60$. There were also no differences in age between those that did

Clinical characteristics of adolescent depression

Corresponding author: Orchard 
and did not meet criteria specifically for a diagnosis of depression, $t(95)=-.50, p=.62$, and no gender difference in those with and without depression, $\chi^{2}(1)=1.70, p=.19$.

\section{Symptom Measures}

The mean scores of the whole sample on adolescent and caregiver reported symptoms of anxiety and depression in the referred adolescents are shown in Table 2. Adolescent selfreported anxiety and depression were highly correlated (Table 2). Parent reported MFQ and adolescent reported MFQ were significantly difference $t(72)=5.96, p<.001$. The mean adolescents MFQ score was well above the suggested cut-offs for clinically meaningful symptom scores (i.e. cut offs of 29 and 27, respectively Burleson Daviss et al., 2006; Wood, Kroll, Moore, \& Harrington, 1995). In contrast the mean parent MFQ score was below the suggested cut-off of 27 for parent-reported scores (Burleson Daviss et al., 2006). Surprisingly there was also no significant association between adolescent and parent reported symptoms of adolescent depression (see Table 2).

Adolescents who met diagnostic criteria for any disorder reported significantly higher MFQ scores; $(M=40.36, M=33.30 ; t(86)=-2.52, p=.013)$. Caregivers of adolescents with and without diagnoses did not differ in their MFQ ratings (with diagnosis, $M=25.00$, without diagnosis, $M=23.13 ; t(75)=-.64, p=.52)$.

\section{Caregiver's symptoms}

Caregivers completed self-reports of their own symptoms of depression, anxiety and stress. According to the DASS, $27 \%$ of caregivers had depression scores that fell in the severe to extremely severe range, $33 \%$ had anxiety scores that fell within a severe to extremely severe range. $36 \%$ had stress scores that fell within a severe to extremely severe range. Mean caregiver DASS scores did not differ between caregivers whose adolescent did and did not meet criteria for any diagnoses $(p>.05)$, or between caregivers of an adolescent

Clinical characteristics of adolescent depression

Corresponding author: Orchard 
with and without a diagnosis of depression $(p>.05)$. As expected, there were significant positive correlations between caregiver's own symptoms and those they reported for their child (Table 2).

[Insert Table 2]

\section{Discussion}

This study assessed the diagnoses and symptoms of adolescents referred for depression to a UK routine public mental health service for children and adolescents. The majority of referred adolescents did not meet criteria for depression (57\%); 23\% of the sample met diagnostic criteria for an alternate mental health disorder, and $34 \%$ did not meet criteria for any disorder. However adolescents who did not meet diagnostic criteria for any disorder reported MFQ scores well above the cut-off for clinically meaningful scores and a significant proportion reported suicidal ideation.

Treatment guidelines for depression focus on those with the disorder and services are typically commissioned and structured to provide treatment for young people with a diagnosis. Despite this, most child and adolescent mental health teams do not conduct structured diagnostic interviews and may therefore not distinguish between those with a diagnosis of depression and those who do not meet diagnostic criteria. This raises questions about what treatment or intervention should be offered to young people who present with symptoms of depression but do not meet criteria for a diagnosis. Longitudinal research indicates that adolescents who have a sub-clinical depression are at increased risk of developing a depressive disorder (Klein, Shankman, Lewinsohn, \& Seeley, 2009; Lewinsohn, Solomon, Seeley, \& Zeiss, 2000) and are likely to experience continued impairment 
(Fergusson, Horwood, Ridder, \& Beautrais, 2005) if treatment is not provided. This could therefore present an opportunity to potentially prevent depression from developing for these young people if input was provided at an earlier stage. Although individuals with sub-clinical levels of depression are likely to still benefit from treatment (e.g. Cuijpers, Koole, van Dijke, Roca, Li, \& Reynolds,2014), there are not currently evidence-based interventions for this population.

The present sample was highly comorbid; of those who met criteria for a depressive disorder 55\% had a comorbid anxiety disorder. These data are consistent with reports from different countries and presenting to different types of services (Angold \& Costello, 1993; Costello et al., 2006; Ford et al., 2003). In this sample externalizing problems were infrequent, however, this may be an artefact of recruiting from a specialist service for anxiety and depression. Therefore young people with externalizing problems (and low mood or anxiety) may be more likely to be referred to a different care pathway where their externalising symptoms may 'overshadow' internalising symptoms.

Of the adolescents who met criteria for a depressive disorder, the most common symptoms were low mood (or irritability), cognitive and sleep disturbances, suicidal thoughts and negative self-perceptions. Rates of suicidal ideation were very high ( $86 \%$ of those diagnosed with depression, $35 \%$ of those with no diagnosis) and in excess of those reported in recent RCTs (TADS, 29.2\%; TADS team, 2005; TORDIA, 58.5\%; Brent et al., 2008; IMPACT 61\%; Goodyer et al., 2015). This is even more striking given that our sample excludes young people identified as at immediate risk who were given a different assessment and are not included in this study.

We do not know if this level of risk is typical of UK public mental health services for children and adolescents. However, the theme of self harm is consistent with a recent 
qualitative study of 77 depressed adolescents. Young people's accounts of their experiences highlighted 'anger and violence towards self and others' (Midgley et al., 2015). This level of risk may also reflect a referral and selection process in UK child and adolescent mental health services that prioritises young people who disclose thoughts of self harm and suicide. This would be clinically understandable and may be the best way to prioritise scarce resources. However, the extremely high rate of suicidal ideation in the sample suggests that other adolescents with clinically significant symptoms of depression who do not disclose or declare suicidal thoughts, may not be referred for treatment and assessment. Such high levels of risk also present a significant emotional burden and workload for clinicians which may not be acknowledged in commissioning, job planning or staff support services.

Young people and their caregivers presented strikingly different descriptions of the severity of young people's anxiety and depression symptoms. Using parent and young person versions of the same self-report questionnaires, caregivers reported that their child's symptoms were significantly and substantially lower than the child themselves reported. Within dyads there was no association between parent reported severity and the young person's own rating of severity. These data are different from research on child anxiety. Niditch and Varela (2011) found that among children aged 7-13 years, parents under-reported symptoms of anxiety in the younger children, and over-reported symptoms for the older children. It has also been found that there is greater agreement for observable symptoms in child anxiety (Comer \& Kendall, 2004). Observational and questionnaire based studies have shown that depressed adolescents and their parents experience high rates of conflict (McLeod, Weisz, \& Wood, 2007), which may result in poor communication between adolescents and their parents. This may partly explain why a large proportion of young people with mental health problems do not access health services (Green et al., 2005).

Clinical characteristics of adolescent depression

Corresponding author: Orchard 
A key strength of this study is that participants and caregivers were recruited from a routine mental health service for children and adolescents. A very large proportion of those invited to take part gave consent i.e. just below $100 \%$. However, not all referrals could be invited into the study. Adolescents were excluded both at the point of entry to the service (if symptoms appeared mild or inappropriate), and at the assessment stage (if an urgent assessment or alternate intervention was needed). This limitation to recruitment was enforced by the service leaders in order to ensure patients were given the appropriate clinical care. A second strength was that diagnostic assessments were conducted by trained and reliable assessors using standardised clinical interviews and well-validated self-report measures and young people and their caregivers were assessed separately to increase reliability (Costello, 1989). This assessment process is not typically part of routine clinical assessment in public child and adolescent mental health services in the UK but as it is recommended in the UK guidelines for assessment of depression in young people (NICE, 2015) it may warrant replication in other settings. However, diagnostic interviews should still be used as part of a multimethod-multistage approach to assessment (e.g. Rutter, Tizard \& Whitmore, 1970), which includes a range of both self-report measurements and clinical interviews.

Caution is needed when interpreting these data. It is unclear how well these data can be generalized to other services. Commissioning arrangements and context may mean that service user characteristics are very different. Of particular concern is that in this study participants were predominantly White British and from relatively high socioeconomic backgrounds. Thus the service users did not reflect the demographics of the local community, which suggests a clear problem with access.

These important caveats highlight the pressing need for similar data to be collected more systematically in routine public child and adolescent mental health services. Planning,

Clinical characteristics of adolescent depression

Corresponding author: Orchard 
developing and delivering effective, accessible and comprehensive mental health services is a high priority on the national political agenda (Department of Health, 2015, 2016). Accurate information about the basic characteristics and needs of service users is an essential tool for decision making about service transformation and investment to improve mental health care for children and young people.

\section{Conclusion}

Depression was diagnosed in 43 of 100 adolescents aged 12-18 years referred for depression to a routine public child and adolescent mental health service in England. Adolescents who met criteria for a depressive disorder most commonly experienced symptoms of low mood (or irritability), cognitive and sleep disturbances, suicidal thoughts and negative self-perceptions. There was a very high level of suicidal ideation in young people with and without a diagnosis of depression. This may reflect a referral bias whereby depressed young people who do not describe suicidal ideation are not prioritised or signposted to local services, and may not be able to access assessment or treatment.

Clinical characteristics of adolescent depression

Corresponding author: Orchard 


\section{Acknowledgements}

Research Funding: Faith Orchard was funded by a PhD studentship at the University of Reading.

Conflicts of Interest: The authors have declared that they have no competing or potential conflicts of interest.

Contributorship: Faith Orchard and Shirley Reynolds designed the project. Faith Orchard recruited participants and collected the data; she also had full access to all the data in the study and takes responsibility for the integrity of the data and the accuracy of the data analysis. Laura Pass and Tamsin Marshall coordinated the recruitment and assessment supervision of clinical participants. All authors share responsibility for the final version of the work submitted.

The authors would also like to thank the participating families and the staff at the Anxiety and Depression Pathway at the University of Reading and Berkshire Healthcare NHS Foundation Trust, in particular Rachael Adams, Sarah Armitage, Sophie Boothe, Kirsten Corden, Sue Cruddace, Jeni Fisk, Poppy Jones, Ambreen Masih, Jayne Morriss, Emily Nobes, Louise Noble, Alice Parker, Jasmine Raw, Hannah Rogers, Holly Tricker, Nick White and Lucy Willetts for their help collecting and coding data.

Clinical characteristics of adolescent depression

Corresponding author: Orchard 


\section{References}

Angold, A., \& Costello, E. (1993). Depressive comorbidity in children and adolescents. American Journal of Psychiatry, 150, 1779-1791.

Angold, A., Costello, E. J., \& Erkanli, A. (1999). Comorbidity. Journal of Child Psychology and Psychiatry, 40(01), 57-87.

Antony, M. M., Bieling, P. J., Cox, B. J., Enns, M. W., \& Swinson, R. P. (1998). Psychometric properties of the 42-item and 21-item versions of the Depression Anxiety Stress Scales in clinical groups and a community sample. Psychological assessment, 10(2), 176.

American Psychiatric Association (1994). Diagnostic and statistical manual of mental disorders. American Psychiatric Association. Washington, DC, 471-475.

American Psychiatric Association (2013). Diagnostic and Statistical Manual of Mental Disorders (DSM-5®): American Psychiatric Pub.

Brent, D., Emslie, G., Clarke, G., Wagner, K. D., Asarnow, J. R., Keller, M., . . Abebe, K. (2008). Switching to another SSRI or to venlafaxine with or without cognitive behavioral therapy for adolescents with SSRI-resistant depression: the TORDIA randomized controlled trial. Jama, 299(8), 901-913.

Bridge, J. A., Goldstein, T. R., \& Brent, D. A. (2006). Adolescent suicide and suicidal behavior. Journal of Child Psychology and Psychiatry, 47(3-4), 372-394.

Burleson Daviss, W., Birmaher, B., Melhem, N., Axelson, D., Michaels, S., \& Brent, D. (2006). Criterion validity of the Mood and Feelings Questionnaire for depressive episodes in clinic and non-clinic subjects. Journal of Child Psychology and Psychiatry, 47(9), 927-934. doi: 10.1111/j.1469-7610.2006.01646.x

Clinical characteristics of adolescent depression

Corresponding author: Orchard 
Chorpita, B. F., Moffitt, C. E., \& Gray, J. (2005). Psychometric properties of the Revised Child Anxiety and Depression Scale in a clinical sample. Behaviour Research and Therapy, 43(3), 309-322.

Chorpita, B. F., Yim, L., Moffitt, C., Umemoto, L. A., \& Francis, S. E. (2000). Assessment of symptoms of DSM-IV anxiety and depression in children: A revised child anxiety and depression scale. Behaviour Research and Therapy, 38(8), 835-855.

Comer, J. S., \& Kendall, P. C. (2004). A symptom-level examination of parent-child agreement in the diagnosis of anxious youths. Journal of the American Academy of Child \& Adolescent Psychiatry, 43(7), 878-886.

Costello, E. J. (1989). Child psychiatric disorders and their correlates: A primary care pediatric sample. Journal of the American Academy of Child \& Adolescent Psychiatry, 28(6), 851-855.

Costello, E. J., Erkanli, A., \& Angold, A. (2006). Is there an epidemic of child or adolescent depression? Journal of Child Psychology and Psychiatry, 47(12), 1263-1271.

Cuijpers, P., Koole, S. L., van Dijke, A., Roca, M., Li, J., \& Reynolds, C. F. (2014). Psychotherapy for subclinical depression: meta-analysis. The British Journal of Psychiatry, 205(4), 268-274.

Department of Health, Taskforce of Children and Young People's Mental Health. (2015).

Future in mind: promoting, protecting and improving our children's and young people's mental health wellbeing (Report No. 02939). Retrieved from https://www.gov.uk/government/uploads/system/uploads/attachment_data/file/414024 /Childrens_Mental_Health.pdf

Department of Health, Taskforce of Children and Young People's Mental Health. (2016). Children and young people's mental health services baselining report (Report No.

Clinical characteristics of adolescent depression

Corresponding author: Orchard 
04894). Retrieved from https://www.england.nhs.uk/mentalhealth/wpcontent/uploads/sites/29/2015/08/nhse-camhs-baselining-summary1.pdf

Dunn, V., \& Goodyer, I. M. (2006). Longitudinal investigation into childhood-and adolescence-onset depression: psychiatric outcome in early adulthood. The British Journal of Psychiatry, 188(3), 216-222.

Essau, C. A., \& Chang, W. C. (2009). Epidemiology, comorbidity, and course of adolescent depression. Treatment of adolescent depression, 4-25.

Fergusson, D. M., Boden, J. M., \& Horwood, L. J. (2007). Recurrence of major depression in adolescence and early adulthood, and later mental health, educational and economic outcomes. The British Journal of Psychiatry, 191(4), 335-342.

Fergusson, D. M., Horwood, L. J., Ridder, E. M., \& Beautrais, A. L. (2005). Subthreshold depression in adolescence and mental health outcomes in adulthood. Archives of General Psychiatry, 62(1), 66-72.

Fergusson, D. M., \& Woodward, L. J. (2002). Mental health, educational, and social role outcomes of adolescents with depression. Archives of General Psychiatry, 59(3), 225231.

Ford, T., Goodman, R., \& Meltzer, H. (2003). The British child and adolescent mental health survey 1999: the prevalence of DSM-IV disorders. Journal of the American Academy of Child \& Adolescent Psychiatry, 42(10), 1203-1211.

Franko, D. L., Striegel-Moore, R. H., Thompson, D., Schreiber, G. B., \& Daniels, S. R. (2005). Does adolescent depression predict obesity in black and white young adult women? Psychological medicine, 35(10), 1505-1513.

Clinical characteristics of adolescent depression

Corresponding author: Orchard 
Goodyer, I., Wright, C., \& Altham, P. (1990). The friendships and recent life events of anxious and depressed school-age children. The British Journal of Psychiatry, 156(5), 689-698.

Gotlib, I. H., Lewinsohn, P. M., \& Seeley, J. R. (1998). Consequences of depression during adolescence: marital status and marital functioning in early adulthood. Journal of Abnormal Psychology, 107(4), 686.

Green, H., McGinnity, Á., Meltzer, H., Ford, T., \& Goodman, R. (2005). Mental health of children and young people in Great Britain, 2004: Palgrave Macmillan Basingstoke.

Halperin, J. M., Rucklidge, J. J., Powers, R. L., Miller, C. J., \& Newcorn, J. H. (2010). Childhood CBCL bipolar profile and adolescent/young adult personality disorders: A 9-year follow-up. Journal of Affective Disorders, 130, 155-161.

Harrington, R., \& Dubicka, B. (2001). Natural History of Mood Disorders in Children and Adolescents. In I. Goodyer (Ed.), The Depressed Child and Adolescent (pp. 311-343). Cambridge: Cambridge University Press.

Harrington, R., Fudge, H., Rutter, M., Pickles, A., \& Hill, J. (1991). Adult outcomes of childhood and adolescent depression: II. Links with antisocial disorders. Journal of the American Academy of Child \& Adolescent Psychiatry, 30(3), 434-439.

Hawker, D. S., \& Boulton, M. J. (2000). Twenty years' research on peer victimization and psychosocial maladjustment: a meta-analytic review of cross-sectional studies. Journal of Child Psychology and Psychiatry, 41(4), 441-455.

Hyde, J. S., Mezulis, A. H., \& Abramson, L. Y. (2008). The ABCs of depression: integrating affective, biological, and cognitive models to explain the emergence of the gender difference in depression. Psychological review, 115(2), 291.

Clinical characteristics of adolescent depression

Corresponding author: Orchard 
Kaufman, J., Birmaher, B., Brent, D., Rao, U., Flynn, C., Moreci, P., . . Ryan, N. (1997). Schedule for affective disorders and schizophrenia for school-age children-present and lifetime version (K-SADS-PL): initial reliability and validity data. Journal of the American Academy of Child \& Adolescent Psychiatry, 36(7), 980-988.

Klein, D. N., Shankman, S. A., Lewinsohn, P. M., \& Seeley, J. R. (2009). Subthreshold depressive disorder in adolescents: predictors of escalation to full-syndrome depressive disorders. Journal of the American Academy of Child \& Adolescent Psychiatry, 48(7), 703-710.

Leaf, P. J., Alegria, M., Cohen, P., Goodman, S. H., Horwitz, S. M., Hoven, C. W., . . Regier, D. A. (1996). Mental health service use in the community and schools: Results from the four-community MECA study. Journal of the American Academy of Child \& Adolescent Psychiatry, 35(7), 889-897.

Lewinsohn, P. M., Rohde, P., Klein, D. N., \& Seeley, J. R. (1999). Natural course of adolescent major depressive disorder: I. Continuity into young adulthood. Journal of the American Academy of Child \& Adolescent Psychiatry, 38(1), 56-63.

Lewinsohn, P. M., Rohde, P., Seeley, J. R., Klein, D. N., \& Gotlib, I. H. (2003). Psychosocial functioning of young adults who have experienced and recovered from major depressive disorder during adolescence. Journal of Abnormal Psychology, 112(3), 353.

Lewinsohn, P. M., Solomon, A., Seeley, J. R., \& Zeiss, A. (2000). Clinical implications of" subthreshold" depressive symptoms. Journal of Abnormal Psychology, 109(2), 345.

Lovibond, P. F., \& Lovibond, S. H. (1995). The structure of negative emotional states: Comparison of the Depression Anxiety Stress Scales (DASS) with the Beck

Clinical characteristics of adolescent depression

Corresponding author: Orchard 
Depression and Anxiety Inventories. Behaviour Research and Therapy, 33(3), 335343.

McLeod, B. D., Weisz, J. R., \& Wood, J. J. (2007). Examining the association between parenting and childhood depression: A meta-analysis. Clinical Psychology Review, 27(8), 986-1003.

Midgley, N., Parkinson, S., Holmes, J., Stapley, E., Eatough, V., \& Target, M. (2015). Beyond a diagnosis: The experience of depression among clinically-referred adolescents. Journal of adolescence, 44, 269-279.

NICE. (2015). Depression in children and young people: Identification and management in primary, community and secondary care. London, UK: National Institute for Health and Care Excellence.

Niditch, L. A., \& Varela, R. E. (2011). Mother-child disagreement in reports of child anxiety: Effects of child age and maternal anxiety. Journal of Anxiety Disorders, 25(3), 450455.

Pine, D. S., Cohen, P., Johnson, J. G., \& Brook, J. S. (2002). Adolescent life events as predictors of adult depression. Journal of Affective Disorders, 68(1), 49-57.

Rao, U., Ryan, N. D., Birmaher, B., Dahl, R. E., Williamson, D. E., Kaufman, J., . . Nelson, B. (1995). Unipolar depression in adolescents: clinical outcome in adulthood. Journal of the American Academy of Child \& Adolescent Psychiatry, 34(5), 566-578.

Reynolds, W. M., \& Mazza, J. J. (1999). Assessment of suicidal ideation in inner-city children and young adolescents: Reliability and validity of the Suicide Ideation Questionnaire-Jr. School Psychology Review, 28, 17-30.

Rice, F., Harold, G., \& Thapar, A. (2002). The genetic aetiology of childhood depression: a review. Journal of Child Psychology and Psychiatry, 43(1), 65-79.

Clinical characteristics of adolescent depression

Corresponding author: Orchard 
Rudolph, K. D., \& Klein, D. N. (2009). Exploring depressive personality traits in youth: origins, correlates, and developmental consequences. Dev Psychopathol, 21(4), 11551180.

Rutter, M., Tizard, J., \& Whitmore, K. (eds.) (1970) Education, Health and Behaviour London: Longmans.

Tabachnick, B. G., \& Fidell, L. S. (2007). Experimental designs using ANOVA. Thomson/Brooks/Cole.

TADS. (2005). The treatment for adolescents with depression study (TADS): Demographic and clinical characteristics. Journal of the American Academy of Child \& Adolescent Psychiatry, 44(1), 28-40.

Thapar, A., Collishaw, S., Pine, D. S., \& Thapar, A. K. (2012). Depression in adolescence. The Lancet, 379(9820), 1056-1067.

Tully, E. C., Iacono, W. G., \& McGue, M. (2008). An adoption study of parental depression as an environmental liability for adolescent depression and childhood disruptive disorders. American Journal of Psychiatry, 165(9), 1148-1154.

Weiss, B., \& Garber, J. (2003). Developmental differences in the phenomenology of depression. Development and psychopathology, 15(02), 403-430.

Wood, A., Kroll, L., Moore, A., \& Harrington, R. (1995). Properties of the Mood and Feelings Questionnaire in adolescent psychiatric outpatients: A research note. Journal of Child Psychology and Psychiatry, 36, 327-334.

Clinical characteristics of adolescent depression

Corresponding author: Orchard 
Table 1. Frequencies of DSM-IV criteria depressive symptoms in adolescents that were assessed.

\begin{tabular}{lccc}
\hline Frequency (\%) & $\begin{array}{c}\text { Adolescents } \\
\text { diagnosed with a } \\
\text { depressive disorder } \\
n=43\end{array}$ & $\begin{array}{c}\text { Adolescents } \\
\text { diagnosed with } \\
\text { another DSM-IV } \\
\text { disorder } \\
n=23\end{array}$ & $\begin{array}{c}\text { Adolescents with } \\
\text { no DSM-IV } \\
\text { diagnosis } \\
n=34\end{array}$ \\
\hline Low mood / irritability & 100 & 54 & 62 \\
Anhedonia & 50 & 0 & 9 \\
Suicidal ideation & 76 & 33 & 35 \\
Sleep disturbances & 43 & 33 & 3 \\
Fatigue & 60 & 25 & 15 \\
Eating disturbances & 70 & 25 & 21 \\
Cognitive disturbances & 19 & 17 & 35 \\
Psychomotor changes & 86 & 0 & \\
Negative self-perceptions & & 50 & \\
\hline
\end{tabular}

Clinical characteristics of adolescent depression

Corresponding author: Orchard 
Table 2. Adolescent and caregiver reported symptom measure means and inter-correlations

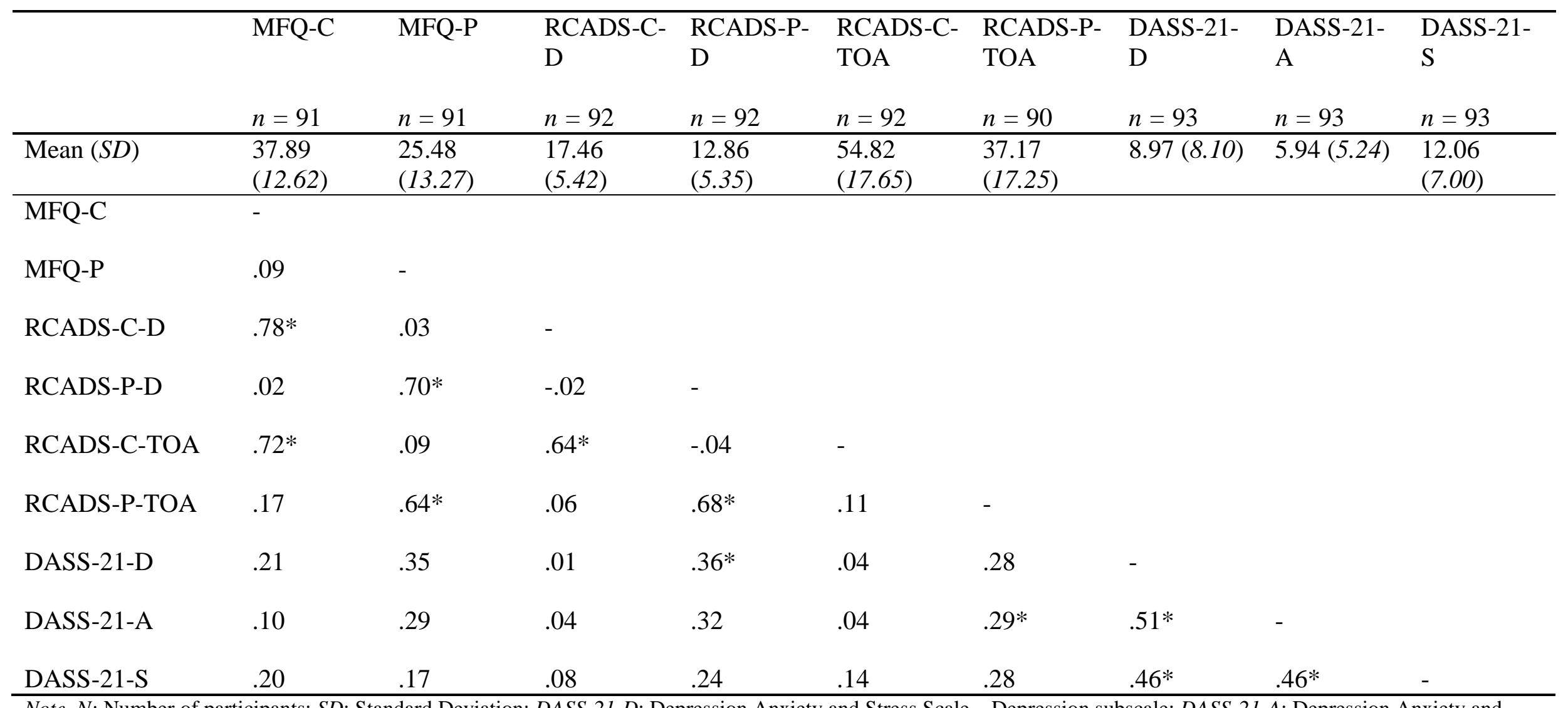

Note. N: Number of participants; $S D$ : Standard Deviation; DASS-21-D: Depression Anxiety and Stress Scale - Depression subscale; DASS-21-A: Depression Anxiety and Stress Scale - Anxiety subscale; DASS-21-S: Depression Anxiety and Stress Scale - Stress subscale; MFQ-C: Mood and Feelings Questionnaire - Child Report; MFQ-P: Mood and Feelings Questionnaire - Parent Report; RCADS-C-D: Revised Child Anxiety and Depression Scale - Child Report on Depression; RCADS-P-D: Revised Child Anxiety and Depression Scale - Parent Report on Depression; RCADS-C-TOA: Revised Child Anxiety and Depression Scale - Child Report on Total Anxiety; RCADS-PTOA: Revised Child Anxiety and Depression Scale - Parent Report on Total Anxiety.

$* p<.001$

Clinical characteristics of adolescent depression

Corresponding author: Orchard 